\title{
Commonly Use of Oral Antibiotics Resistance in Children Aged 1 to 12 years with UTI's a Increasing Problems
}

\author{
Batabyal B ${ }^{1 *}$ and Himanshu ${ }^{2}$ \\ ${ }^{1}$ Research Scholar, OPJS University Churu, Rajasthan, India \\ ${ }^{2}$ Associate Professor, Department of Microbiology, OPJS University, Churu, Rajasthan, \\ India
}

*Corresponding author: Biswajit Batabyal, Research Scholar, OPJS University Churu, Rajasthan, India, Email: biswajit.batabyal@gmail.com

\section{Abstract}

Background: Urinary tract infections (UTIs) are counted among the most common infections in children. Most commonly, members of Enterobacteriacea, particularly urinary pathogenic strains of E. coli and Enterobacter aerogenes are the primary causative organisms of UTIs in different parts of the world. . In spite of the availability and use of the antimicrobial drugs, UTIs caused by bacteria have been showing increasing trends. Antibiotics are a mainstay in the treatment of bacterial infections, though their use is a primary risk factor for the development of antibiotic resistance. Antibiotic resistance is a growing problem in pediatric urology as demonstrated by increased urinary pathogen resistance. The extensive and inappropriate use of antimicrobial agents has invariably resulted in the development of antibiotic resistance which, in recent years, has become a major problem worldwide. Increasing antibiotic resistance among urinary pathogens to commonly prescribed drugs has become a global reality today. Complex pediatric patients with histories of hospitalizations, prior antibiotic exposure, and recurrent UTIs are also at high risk for acquiring UTIs due to extended spectrum beta-lactamase [ESBL] producing organisms. Data regarding the impact of in vitro antibiotic susceptibility testing interpretation on UTI treatment outcomes is lacking. The resistance of bacteria causing urinary tract infection (UTI) to commonly prescribed antibiotics is increasing both in developing as well as in developed countries. Resistance has emerged even to more potent antimicrobial agents.

Objective: The present study was undertaken to report the commonly use of current antibiotic resistance pattern among common bacterial urinary pathogens isolated.

Methodology \& Results: A total of 512 urine samples were collected from out patients of age between 1 to 12 years of both sex of children at Serum Analysis Center Pvt. Ltd. [Referral Laboratory]; Howrah; West Bengal; India between December 2016 to November 2017. The urine samples were cultured on HiCrome UTI Agra media and Eosin Methylene 
Blue Agar media $[\mathrm{EMB}]$ and the bacterial isolates were identified by gram staining and conventional biochemical methods. The bacterial isolates recovered of commonly use of oral antibiotics were tested against Amoxicillin/clavulanate, Cefixime, Cefpodoxime, Cefprozil, Cephalexin and Co-trimoxazole (Trimethoprim/sulfamethoxazole) using Kirby Bauer disk diffusion method according to the current National Committee for Clinical Laboratory Standards (NCCLS) guidelines. Among the 512 urine samples examined [1 to 12 years of children], included 276 (54.0\%) in Male child \& 236 (46.0\%) in Female child and 220 (42.9\%) of urinary pathogens are isolated. The bacteria were isolates 104 (37.7\%) of male child and 116 (49.2\%) of female child. In patient of male child, 50\% of $E$. coli, $34.6 \%$ of Klebsiella pneumoniae, $15.4 \%$ of others gram negative bacilli and $52.0 \%$ Extended- spectrum Beta lactamase [ESBL] stains were isolates. In patient of female child, $72.4 \%$ of E. coli, $20.7 \%$ of Klebsiella pneumoniae , $6.9 \%$ of others gram negative bacilli and 58.7\% Extended- Spectrum Beta lactamase [ESBL] stains were isolates. Resistance rates of E. coli [1 to 12 years of children] isolates were $83.8 \%$ to Amoxicillin/clavulanate, $70.5 \%$ to Cefixime, $89.7 \%$ to Cefpodoxime, $80.8 \%$ to Cefprozil, $89.8 \%$ of Cefalexin and $63.2 \%$ to Co-trimoxazole. Resistance rates of Klebsiella pneumoniae [1 to 12 years of children] isolates were $66.7 \%$ to Amoxicillin/clavulanate, $43.3 \%$ to Cefixime, $90 \%$ to Cefpodoxime, $76.6 \%$ to Cefprozil, $80 \%$ to Cefalexin and $50 \%$ to Co-trimoxazole. Resistance rate of Others gram negative bacilli [ 1 to 12 years of children] isolates were $75 \%$ to Amoxicillin/clavulanate, $33.4 \%$ to Cefixime, $91.6 \%$ to Cefpodoxime, $91.6 \%$ to Cefprozil, $91.6 \%$ to Cefalexin and $41.7 \%$ to Co-trimoxazole.

Conclusion: Increasing antibiotic resistance trends indicate that it is imperative to rationalize the use of antimicrobials in the community and also use these conservatively. It is concluded that the clinical isolates have started developing resistance against commonly use antibiotics due to its irrational and inappropriate use. Continuous surveillance is crucial to monitor the antimicrobial resistance of pathogens. Finally, we suggest that empirical antibiotic selection should be based on knowledge of the local prevalence of bacterial organisms and antibiotic sensitivities rather than on universal guidelines.

Keywords: Urinary Tract Infections; Antibiotic Resistance; Pediatrics; Oral Antibiogram

Abbreviations: UTI: Urinary tract infections; AMR: Antimicrobial resistance; CFU: Colony Forming Unit; ESBL: Extended- spectrum Beta lactamase; NCCLS: National Committee for Clinical Laboratory Standards; EMB: Eosin Methylene Blue; ONPG: Ortho-nitrophenyl beta-D-galactopyranoside; CLSI: Clinical and Laboratory Standards institute.

\section{Introduction}

Urinary tract infections (UTIs) are amongst the most common infections encountered in clinical practice [1]. Acute urinary tract infections are relatively common in children, with 8 percent of girls and 2 percent of boys having at least one episode by seven years of age, and between $30 \%$ and $40 \%$ will have another episode within two years [2-3]. The most common pathogen is Escherichia coli, accounting for approximately 85 percent of urinary tract infections in children (Table 1). Renal parenchymal defects are present in 3 to 15 percent of children within one to two years of their first diagnosed urinary tract infection. Clinical signs and symptoms of a urinary tract infection depend on the age of the child, but all febrile children two to 24 months of age with no obvious cause of infection should be evaluated for urinary tract infection (with the exception of circumcised boys older than 12 months). Evaluation of older children may depend on the clinical presentation and symptoms that 
point toward a urinary source (e.g., leukocyte esterase or nitrite present on dipstick testing; pyuria of at least 10 white blood cells per high-power field and bacteriuria on microscopy).

Urinary tract infections (UTI) are one of the most important causes of morbidity and mortality in the developing countries like India. Several studies has demonstrated that the geographical variability of pathogen occurrence in case of UTI among inpatients and outpatients populations is limited by the predominance of gram-negative species usually Enterobactericeae and particularly E. coli and Enterobacter spp. in various regions of the world [4-5].

Antimicrobial resistance (AMR) is a global growing issue and several reports suggest that it is an increasing problem of phenomenal proportions, affecting both developed and developing countries [6]. AMR is considered as a natural phenomenon for the survival of micro-organism. Therefore, it is imperative to slow the rate of development of AMR to a level that maintains the usefulness of the antimicrobials [6]. Accurate determination of bacterial susceptibility to antibiotics is essential for the successful management of bacterial infections and comparative analysis of antimicrobial agents (Table 1). Public health officials and clinicians monitor drug resistance through appropriate reporting of the results from susceptibility tests and this can be achieved using a number of techniques, including the disk diffusion method, the broth dilution assay, and the $\mathrm{E}$ tests [7]. As antibiotic resistance reduces treatment efficacy, it is a time to consider routine susceptibility testing to guide individual patient treatment and surveillance of antibiotic resistance [8].

\begin{tabular}{|c|c|c|}
\hline Antibiotic & Dosing & Common adverse effects \\
\hline Amoxicillin/clavulanate(Augmentin) & $\begin{array}{c}25 \text { to } 45 \text { mg per kg per day, } \\
\text { divided every 12 hours }\end{array}$ & Diarrhea, nausea/vomiting, rash \\
\hline Cefixime (Suprax) & $\begin{array}{c}\text { 8 mg per kg every 24 hours or } \\
\text { divided every 12 hours }\end{array}$ & Abdominal pain, diarrhea, flatulence, rash \\
\hline Cefpodoxime & $\begin{array}{c}10 \text { mg per kg per day, divided } \\
\text { every 12 hours }\end{array}$ & Abdominal pain, diarrhea, nausea, rash \\
\hline Cefprozil (Cefzil) & $\begin{array}{c}30 \text { mg per kg per day, divided } \\
\text { every 12 hours }\end{array}$ & $\begin{array}{c}\text { Abdominal pain, diarrhea, elevated results on } \\
\text { liver function tests, nausea }\end{array}$ \\
\hline Cephalexin (Keflex) & $\begin{array}{c}25 \text { to } 50 \text { mg per kg per day, } \\
\text { divided every 6 to 12 hours }\end{array}$ & Diarrhea, headache, nausea/vomiting, rash \\
\hline $\begin{array}{c}\text { Trimethoprim/sulfamethoxazole } \\
\text { (Bactrim, Septra) }\end{array}$ & $\begin{array}{c}\text { to } 10 \text { mg per kg per day, divided } \\
\text { every 12 hours }\end{array}$ & Diarrhea, nausea/vomiting, photosensitivity, rash \\
\hline
\end{tabular}

Table 1: Antibiotics Commonly Used to Treat Urinary Tract Infections in Children.

The present study was undertaken to assess the commonly use of current oral antibiotics resistance pattern in the common urinary pathogens isolated in children of age group 1 to 12 years. The risk assessment was also performed to determine the factors responsible for the emergence of commonly use oral antibiotics resistance in common bacterial urinary pathogens.

\section{Materials and Methods}

\section{Study Population, Design and Setting}

The current study was conducted in the Department of Microbiology, Serum Analysis Center Pvt. Ltd. [Referral Laboratory]; Howrah; West Bengal; India; from December 2016 to November 2017.

\section{Patient Evaluation}

A prospective analysis was done on 512 of outpatients. All patients were within ages 1 to 12 of children, comprising of both male and female. All samples received consisted 276 of male child and 236 of female child.

\section{Categories Age Group}

[i] Preschool aged Children: 1to 5 Years. [ii] School aged Children: $>5$ to 12 Years.

\section{Collection of Urine Sample}

Early morning mid-stream urine samples were collected using sterile, wide mouthed container with screw cap tops [9]. 


\section{Open Access Journal of Microbiology \& Biotechnology}

On the urine sample bottles were indicated name, age, sex, and time of collection along with requisition forms
(Table 2).

\begin{tabular}{|c|c|c|c|}
\hline Age Group & Total Population & Male Child & Female Child \\
\hline 1 to 12 years & 512 & $276(54.0 \%)$ & $236(46.0 \%)$ \\
\hline 1 to 5 years & 312 & $170(54.5 \%)$ & $142(45.5 \%)$ \\
\hline > 5 to 12 years & 200 & $106(53.0 \%)$ & $94(47.0 \%)$ \\
\hline
\end{tabular}

Table 2: Different age groups of total sample.

\section{Sample Processing}

A calibrated sterile micron wire loop for the semiquantitative method was used for the plating and it has a $4.0 \mathrm{~mm}$ diameter designed to deliver $0.01 \mathrm{ml}$. A loopful of the well mixed urine sample was inoculated on HiCrome UTI Agar media and EMB [Eosin Methylene Blue] Agar media. The plate was incubated aerobically at $37^{\circ}$ centrigade for overnight. The plates were then examined macroscopically and microscopical for bacterial growth. The bacterial colonies were counted and multiplied by 100 to give an estimate of the number of bacteria present per mililiter of urine. Culture results were interpreted according to the standard criteria and a growth of $>10^{5}$ colony forming unit [CFU] $/ \mathrm{ml}$ was considered as significant bacteriuria [10]. The urine samples were analyzed bacteriological using the methods $[9,11,12]$ (Table 2).

\section{Identification of Isolates}

The isolates were identified using colony morphology, Gram staining, Motility test, Indole test, Citrate test [Simmons Citrate Agar media], Urease test [Urease Agar media $+40 \%$ Urea], Triple Sugar Iron Agar media, ONPG [Ortho-nitrophenyl beta-D-galactopyranoside] and Oxidase test $[9,12]$.

\section{Antimicrobial Susceptibility Testing}

All isolates were tested for antimicrobial susceptibility on Muller Hinton Agar by the standard Bauer et al. disc diffusion method [13] recommended by the Clinical and Laboratory Standards institute (CLSI) [ 14]. Antibiotic agents (disks) were obtained from Hi Media Laboratories,
Pvt. Ltd; Mumbai. Appropriate quality control strains were used to validate the results of the antimicrobial disk. In this section of the study, bacterial isolates recovered of commonly use of oral antibiotics were tested against Amoxicillin/clavulanate $(20 / 10 \mathrm{mcg})$, Cefixime $(5 \mathrm{mcg})$, Cefpodoxime (10 mcg), Cefprozil $(30 \mathrm{mcg}$ ), Cephalexin (30 $\mathrm{mcg})$ and Co-trimoxazole [Trimethoprim/sulfamethoxazole] $(1.25 / 23.75 \mathrm{mcg}) . E$. coli, ATCC 25922, and Pseudomonas aeruginosa, ATCC 27853 was used as quality control strains [12].

\section{Extended-Spectrum Beta-Lactamase (ESBL) Detection by the CLSI Phenotype Method}

The CLSI ESBL confirmatory test with cefotaxime [30mcg] and Cefotaxime/Clavulanic acid [30+10 mcg] were performed for all isolates using the disc diffusion method on Mueller-Hinton Agar plates. Susceptibility test results were interpreted according to criteria established by the CLSI [15].

\section{Results}

For the twelve months of this study, 512 urine samples were received and cultured. There were 276 (54.0\%) male child and $236(46.0 \%)$ female child giving a total of 512 children who enrolled in this study (Table 2). Their age ranged from 1 to 12 years. Among the cultures screened, bacteriuria of $10^{5} \mathrm{cfu} / \mathrm{ml}$ of urine was found in $220(42.9 \%)$ of the samples. A total of $292(57.0 \%)$ of the urine samples were culture negative (Table 3). 104 $(37.7 \%)$ were isolated from male child and $116(49.2 \%)$ from female child (Tables 4-8).

\begin{tabular}{|c|c|c|c|}
\hline Age Group & Total Population & Positive culture & Negative culture \\
\hline 1 to 12 years & 512 & $220(42.9 \%)$ & $292(57.0 \%)$ \\
\hline 1 to 5 years & 312 & $148(47.4 \%)$ & $164(52.6 \%)$ \\
\hline >5 to 12 years & 200 & $72(36.0 \%)$ & $128(64.0 \%)$ \\
\hline
\end{tabular}

Table 3: Prevalence of UTI in different age groups. 


\section{Open Access Journal of Microbiology \& Biotechnology}

\begin{tabular}{|c|c|c|c|c|}
\hline Age Group & $\begin{array}{c}\text { Total Population in } \\
\text { Male child }\end{array}$ & $\begin{array}{c}\text { Positive culture in } \\
\text { Male child }\end{array}$ & $\begin{array}{c}\text { Total Population in } \\
\text { Female child }\end{array}$ & $\begin{array}{c}\text { Positive culture in Female } \\
\text { child }\end{array}$ \\
\hline 1 to 12 years & 276 & $104(37.7 \%)$ & 236 & $116(49.2 \%)$ \\
\hline 1 to 5 years & 170 & $74(43.6 \%)$ & 142 & $74(52.2 \%)$ \\
\hline 5 5 to 12 years & 106 & $30(28.3 \%)$ & 94 & $42(44.7 \%)$ \\
\hline
\end{tabular}

Table 4: Prevalence of UTI in different age groups with Male \& Female child.

\begin{tabular}{|c|c|c|}
\hline Pathogens & Male child [No: 104] & Female child [No.:116] \\
\hline Esch. Coli & $52(50.0 \%)$ & $84(72.4 \%)$ \\
\hline Klebsiella pneumoniae & $36(34.6 \%)$ & $24(20.7 \%)$ \\
\hline Otheras Gram Negative Bacilli & $16(15.4 \%)$ & $8(6.9 \%)$ \\
\hline ESBL Stain & $54(52.0 \%)$ & $68(58.7 \%)$ \\
\hline
\end{tabular}

Table 5: Prevalence of pathogens isolated on urine culture with age group of 1 to 12 years.

\begin{tabular}{|c|c|c|}
\hline Pathogens & Male child [No.: 74] & Female child [No.: 74] \\
\hline Esch. Coli & $36(48.6 \%)$ & $54(73.0 \%)$ \\
\hline Klebsiella pneumoniae & $26(35.2 \%)$ & $14(18.9 \%)$ \\
\hline Otheras Gram Negative Bacilli & $12(16.2 \%)$ & $06(8.1 \%)$ \\
\hline ESBL Stain & $40(54.0 \%)$ & $44(59.5 \%)$ \\
\hline
\end{tabular}

Table 6: Prevalence of pathogens isolated on urine culture with age group of 1 to 5 years.

\begin{tabular}{|c|c|c|}
\hline Pathogens & Male child [No.: 30] & Female child [No.: 42] \\
\hline Esch. Coli & $16(53.3 \%)$ & $30(71.4 \%)$ \\
\hline Klebsiella pneumoniae & $10(33.3 \%)$ & $10(23.8 \%)$ \\
\hline Otheras Gram Negative Bacilli & $04(13.4 \%)$ & $02(4.8 \%)$ \\
\hline ESBL Stain & $14(46.7 \%)$ & $24(57.2 \%)$ \\
\hline
\end{tabular}

Table 7: Prevalence of pathogens isolated on urine culture with age group of $>5$ to 12 years.

Total Isolates: 136

\begin{tabular}{|c|c|c|c|c|}
\hline Antibiotics & R (No.) & R (\%) & S (No.) & S (\%) \\
\hline Amoxicillin/Clavulanic acid & 114 & 83.8 & 22 & 16.2 \\
\hline Trimethoprim/Sulfamethoxazole & 86 & 63.2 & 50 & 36.8 \\
\hline Cefixime & 96 & 70.5 & 40 & 29.5 \\
\hline Cefpodoxime & 122 & 89.7 & 14 & 10.3 \\
\hline Cefprozil & 110 & 80.8 & 26 & 19.2 \\
\hline Cefalexin & 122 & 89.8 & 14 & 10.2 \\
\hline
\end{tabular}

Table 8: Percentage of Resistant \& Susceptibility of isolated Escherichia coli to tested commonly use of oral antibiotics: [1 TO 12 YEARS]. 


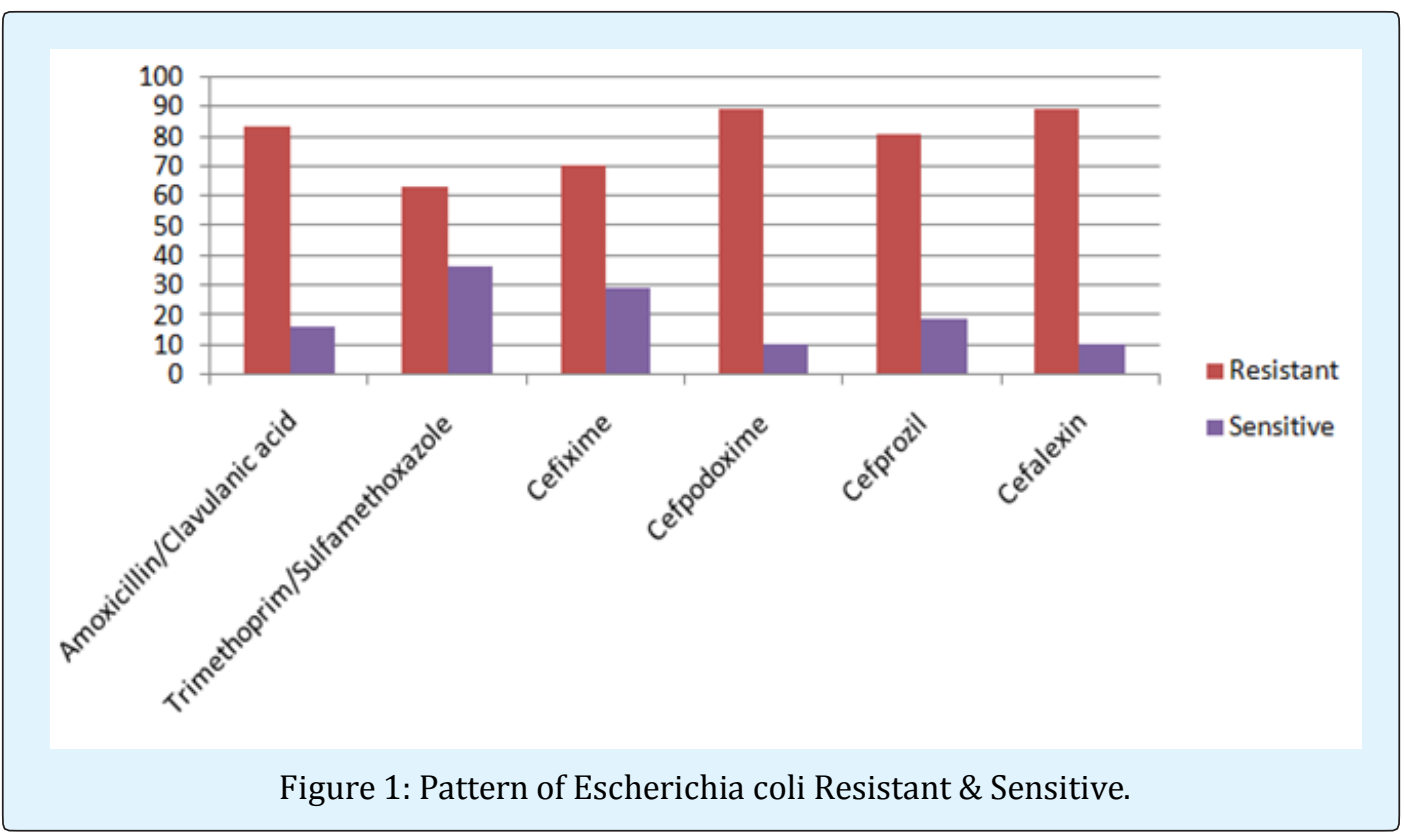

Total Isolates: 60

\begin{tabular}{|c|c|c|c|c|}
\hline Antibiotics & R (No.) & R (\%) & S (No.) & S (\%) \\
\hline Amoxicillin/Clavulanic acid & 40 & 66.7 & 20 & 33.3 \\
\hline Trimethoprim/Sulfamethoxazole & 30 & 50.0 & 30 & 50.0 \\
\hline Cefixime & 26 & 43.3 & 34 & 56.7 \\
\hline Cefpodoxime & 54 & 90.0 & 06 & 10.0 \\
\hline Cefprozil & 46 & 76.6 & 14 & 23.4 \\
\hline Cefalexin & 48 & 80.0 & 12 & 20.0 \\
\hline
\end{tabular}

Table 9: Percentage of Resistant \& Susceptibility of isolated Klebsiella pneumoniae to tested commonly use of oral antibiotics: [1 T0 12 YEARS].

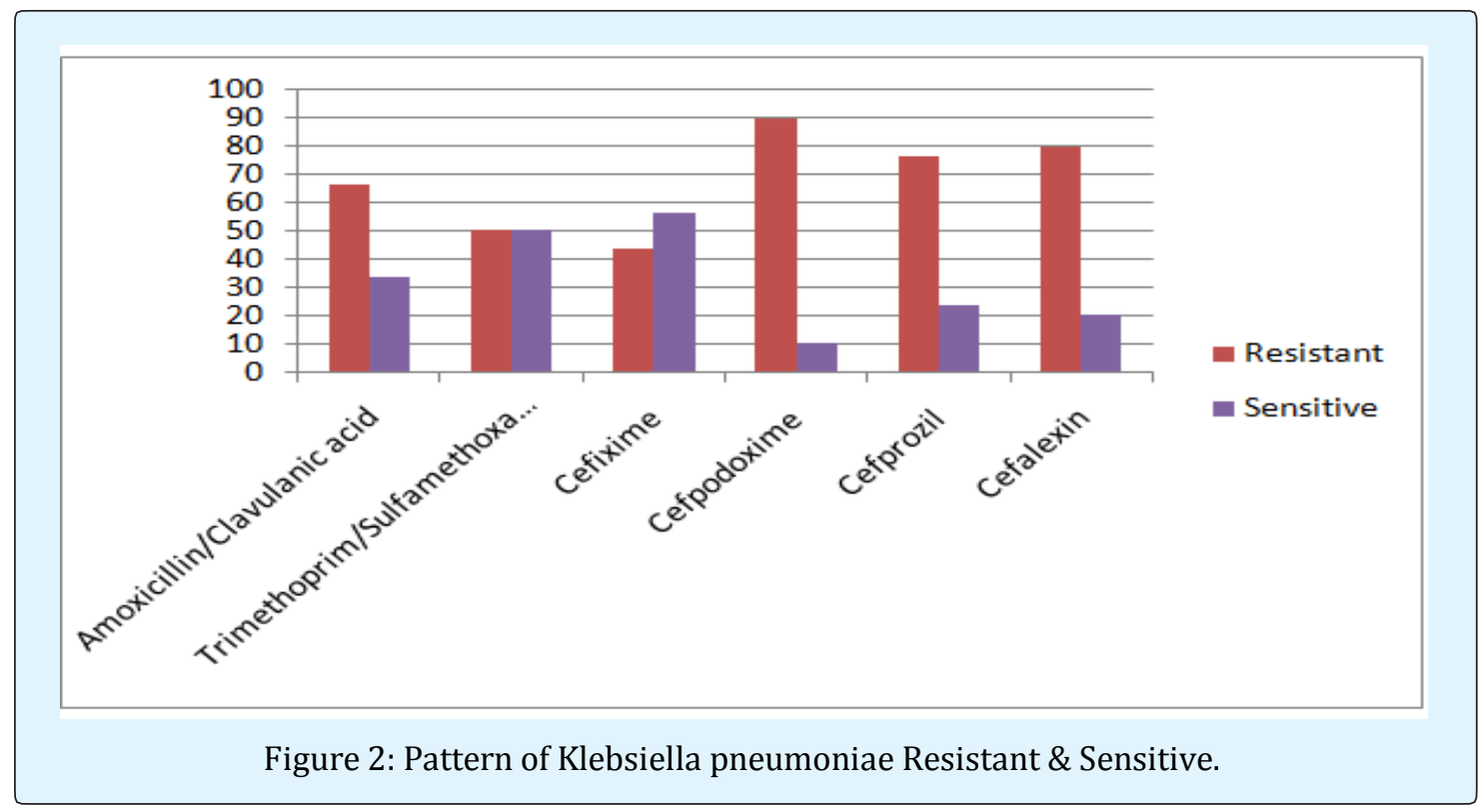


Total Isolates: 24

\begin{tabular}{|c|c|c|c|c|}
\hline Antibiotics & R (No.) & R (\%) & S (No.) & S (\%) \\
\hline Amoxicillin/Clavulanic acid & 18 & 75.0 & 06 & 25.0 \\
\hline Trimethoprim/Sulfamethoxazole & 10 & 41.7 & 14 & 58.3 \\
\hline Cefixime & 08 & 33.4 & 16 & 66.6 \\
\hline Cefpodoxime & 22 & 91.6 & 02 & 08.4 \\
\hline Cefprozil & 22 & 91.6 & 02 & 08.4 \\
\hline Cefalexin & 22 & 91.6 & 02 & 08.4 \\
\hline
\end{tabular}

Table 10: Percentage of Resistant \& Susceptibility of isolated Others Gram Negative Bacilli to tested commonly use of oral antibiotics: [1 T0 12 YEARS].

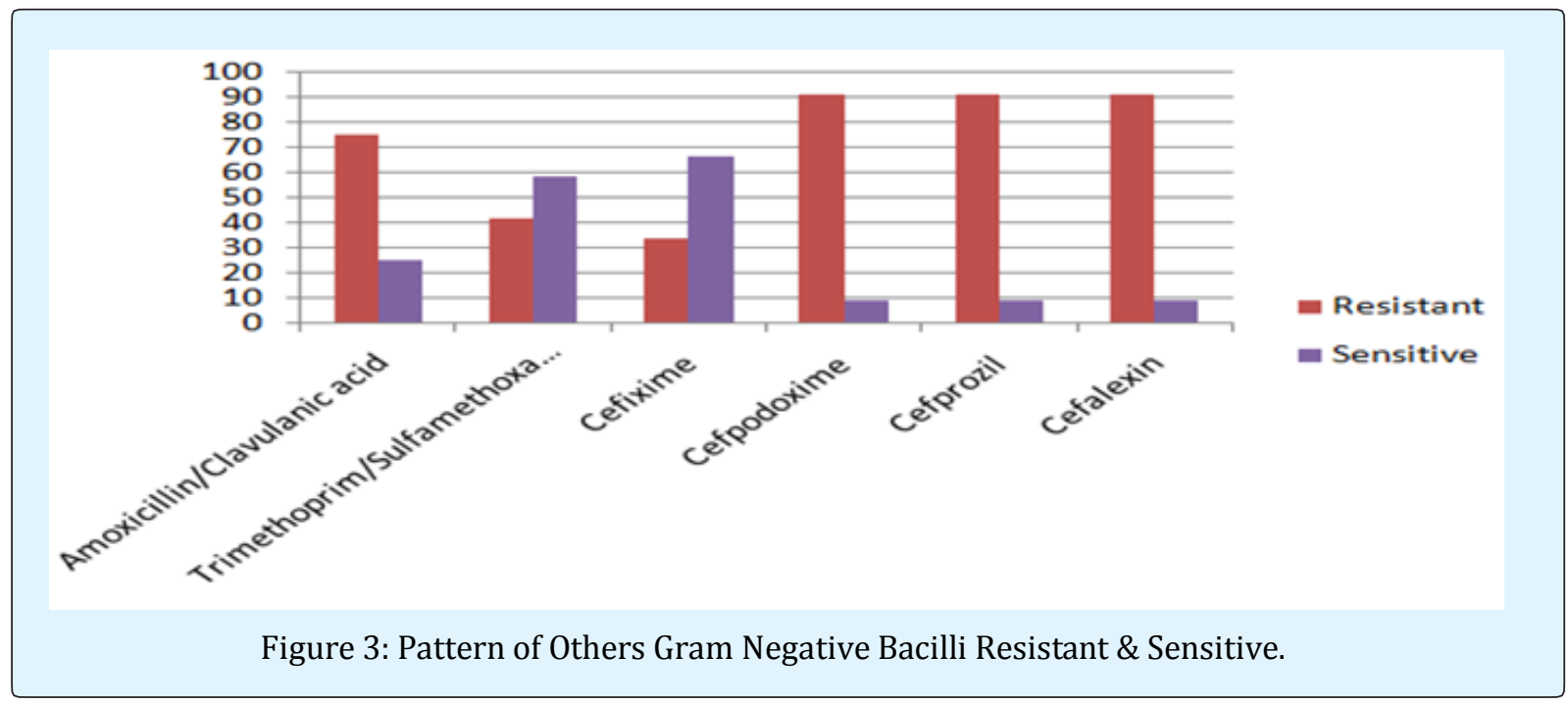

Total Isolates: 90

\begin{tabular}{|c|c|c|c|c|}
\hline Antibiotics & R (No.) & R (\%) & S (No.) & S (\%) \\
\hline Amoxicillin/Clavulanic acid & 80 & 88.9 & 10 & 11.1 \\
\hline Trimethoprim/Sulfamethoxazole & 58 & 64.4 & 32 & 35.6 \\
\hline Cefixime & 62 & 68.8 & 28 & 31.2 \\
\hline Cefpodoxime & 80 & 88.8 & 10 & 11.2 \\
\hline Cefprozil & 70 & 77.8 & 20 & 22.2 \\
\hline Cefalexin & 82 & 91.1 & 08 & 08.9 \\
\hline
\end{tabular}

Table 11: Percentage of Resistant \& Susceptibility of isolated Escherichia coli to tested commonly use of oral antibiotics: [1 TO 5 YEARS]. 


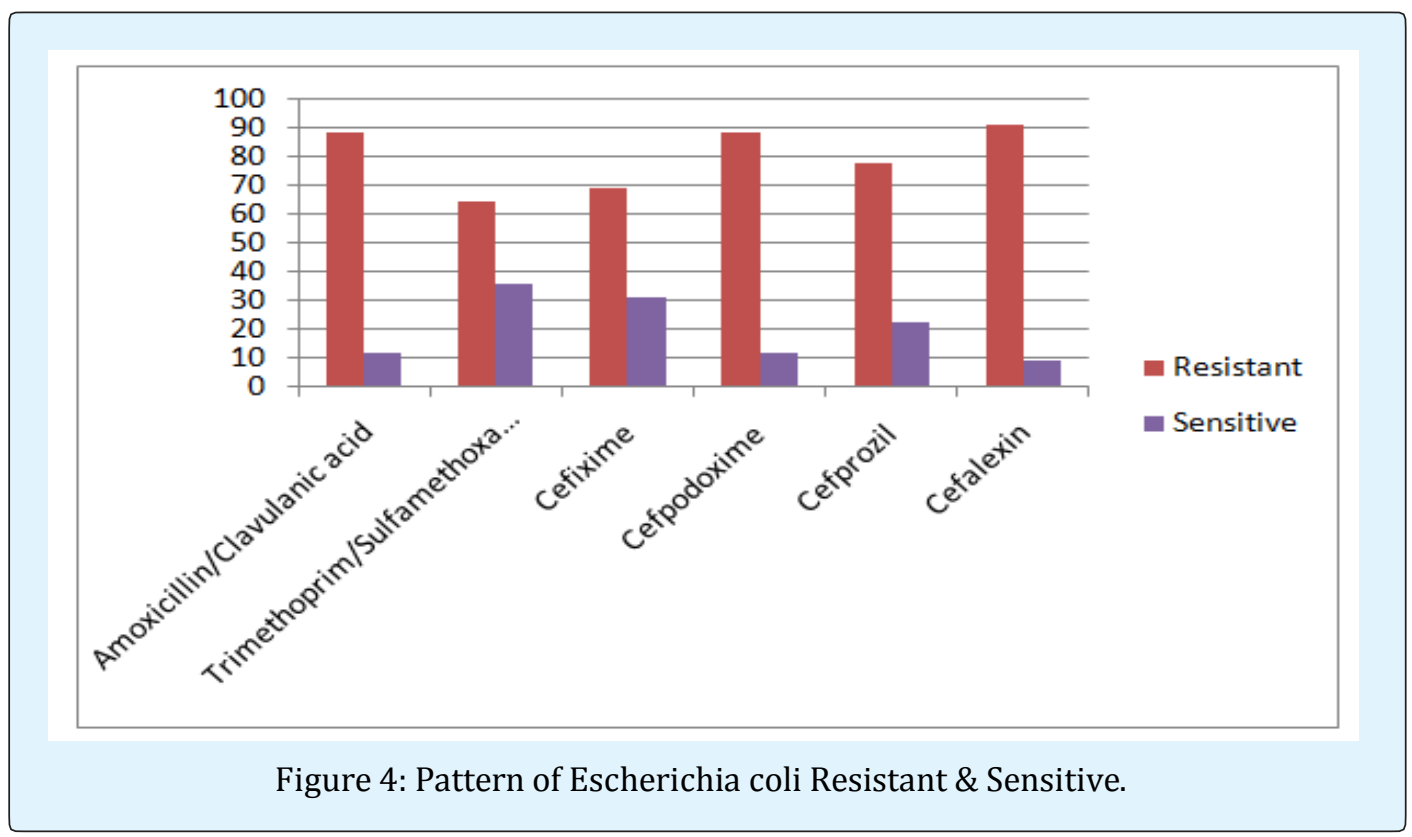

Total Isolates: 40

\begin{tabular}{|c|c|c|c|c|}
\hline Antibiotics & R (No.) & R (\%) & S (No.) & S (\%) \\
\hline Amoxicillin/Clavulanic acid & 30 & 75.0 & 10 & 25.0 \\
\hline Trimethoprim/Sulfamethoxazole & 20 & 50.0 & 20 & 50.0 \\
\hline Cefixime & 20 & 50.0 & 20 & 50.0 \\
\hline Cefpodoxime & 36 & 90.0 & 04 & 10.0 \\
\hline Cefprozil & 34 & 85.0 & 06 & 15.0 \\
\hline Cefalexin & 34 & 85.0 & 06 & 15.0 \\
\hline
\end{tabular}

Table 12: Percentage of Resistant \& Susceptibility of isolated Klebsiella pneumoniae to tested commonly use of oral antibiotics: [1 TO 5 YEARS].

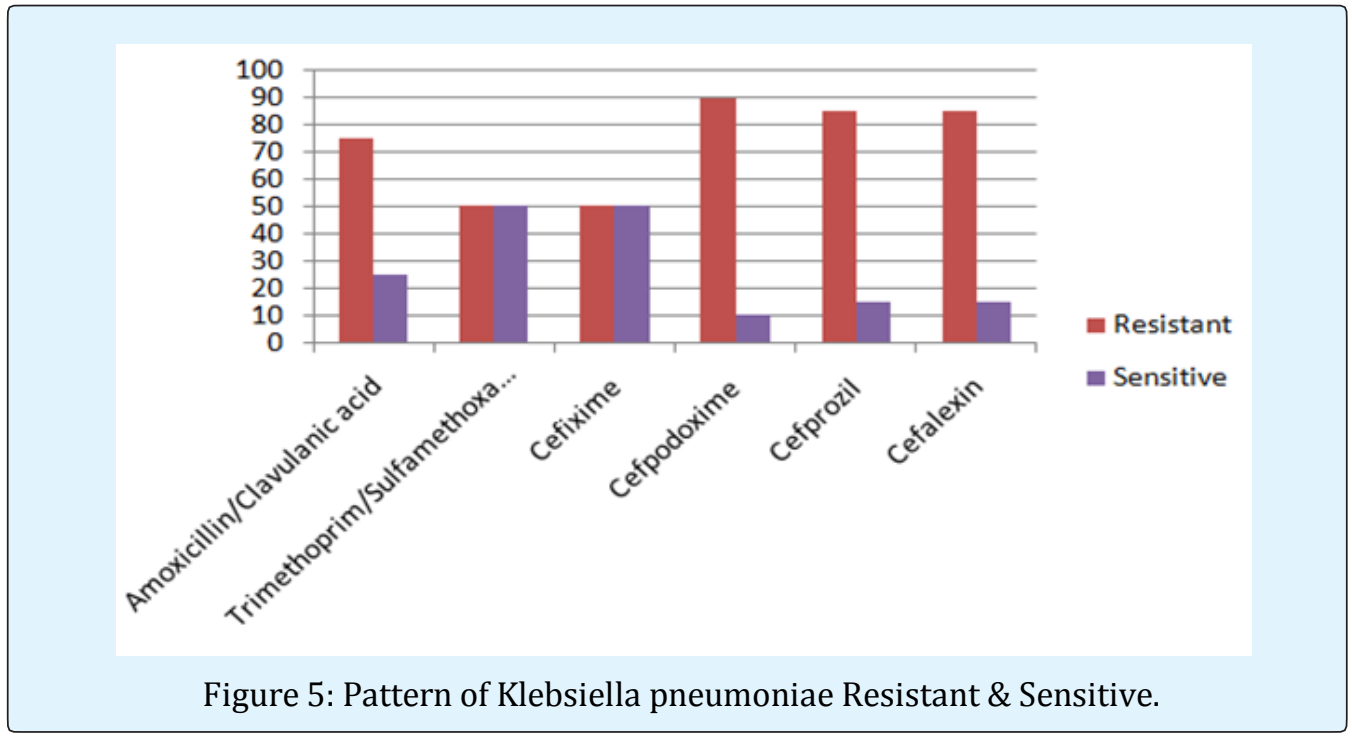


Total Isolates: 18

\begin{tabular}{|c|c|c|c|c|}
\hline Antibiotics & R (No.) & R (\%) & S (No.) & S (\%) \\
\hline Amoxicillin/Clavulanic acid & 14 & 77.8 & 04 & 22.2 \\
\hline Trimethoprim/Sulfamethoxazole & 08 & 44.5 & 10 & 55.5 \\
\hline Cefixime & 07 & 38.8 & 11 & 61.2 \\
\hline Cefpodoxime & 16 & 88.8 & 02 & 11.2 \\
\hline Cefprozil & 16 & 88.8 & 02 & 11.2 \\
\hline Cefalexin & 16 & 88.8 & 02 & 11.2 \\
\hline
\end{tabular}

Table 13: Percentage of Resistant \& Susceptibility of isolated Others Gram Negative Bacilli to tested commonly use of oral antibiotics: [1 T0 5 YEARS].

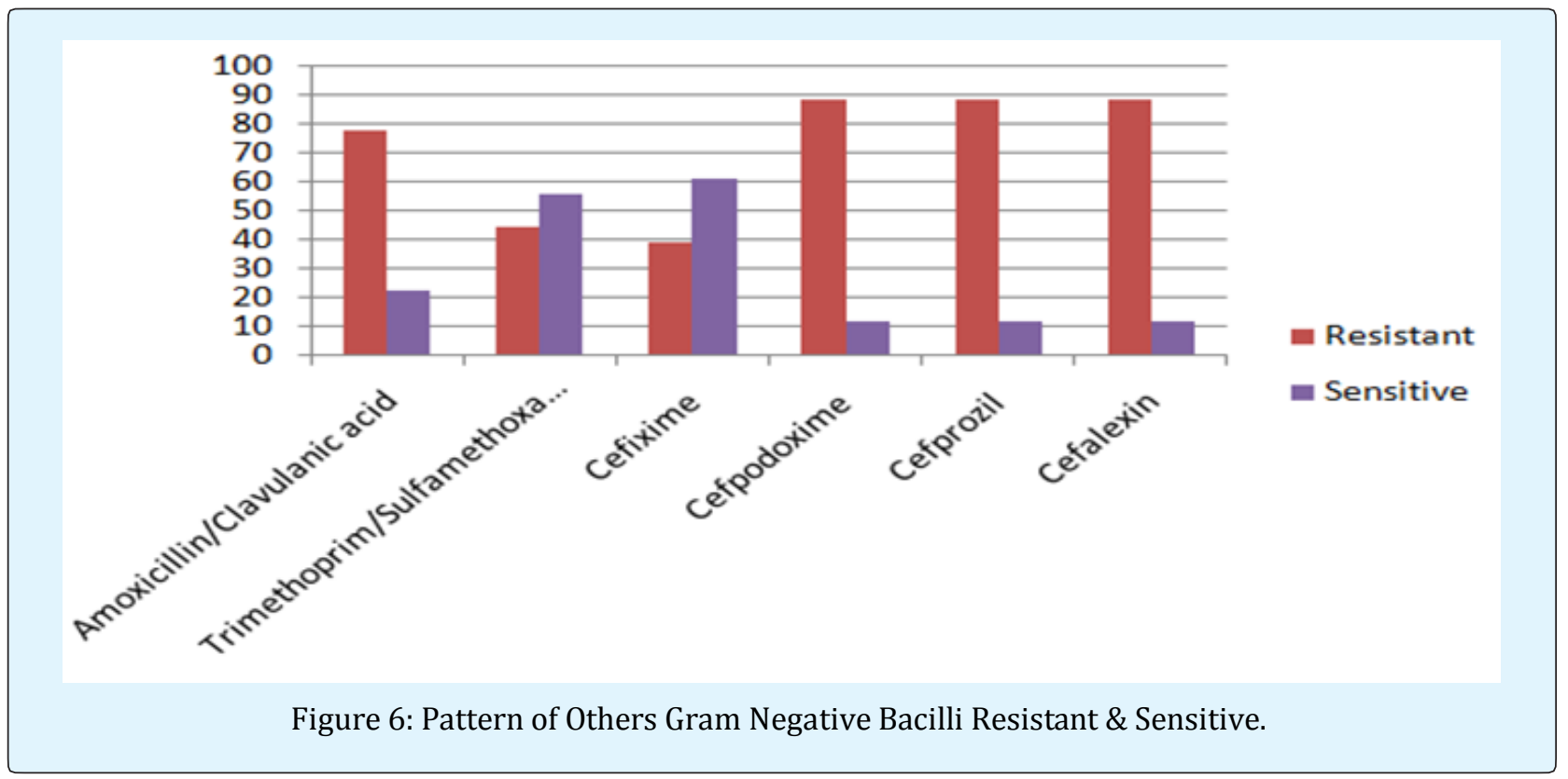

Total Isolates: 46

\begin{tabular}{|c|c|c|c|c|}
\hline Antibiotics & R (No.) & R (\%) & S (No.) & S (\%) \\
\hline Amoxicillin/Clavulanic acid & 34 & 74.0 & 12 & 26.0 \\
\hline Trimethoprim/Sulfamethoxazole & 28 & 60.8 & 18 & 39.2 \\
\hline Cefixime & 34 & 74.0 & 12 & 26.0 \\
\hline Cefpodoxime & 42 & 91.3 & 04 & 08.7 \\
\hline Cefprozil & 40 & 87.0 & 06 & 13.0 \\
\hline Cefalexin & 40 & 87.0 & 06 & 13.0 \\
\hline
\end{tabular}

Table 14: Percentage of Resistant \& Susceptibility of isolated Escherichia coli to tested commonly use of oral antibiotics: [>5 TO 12 YEARS]. 


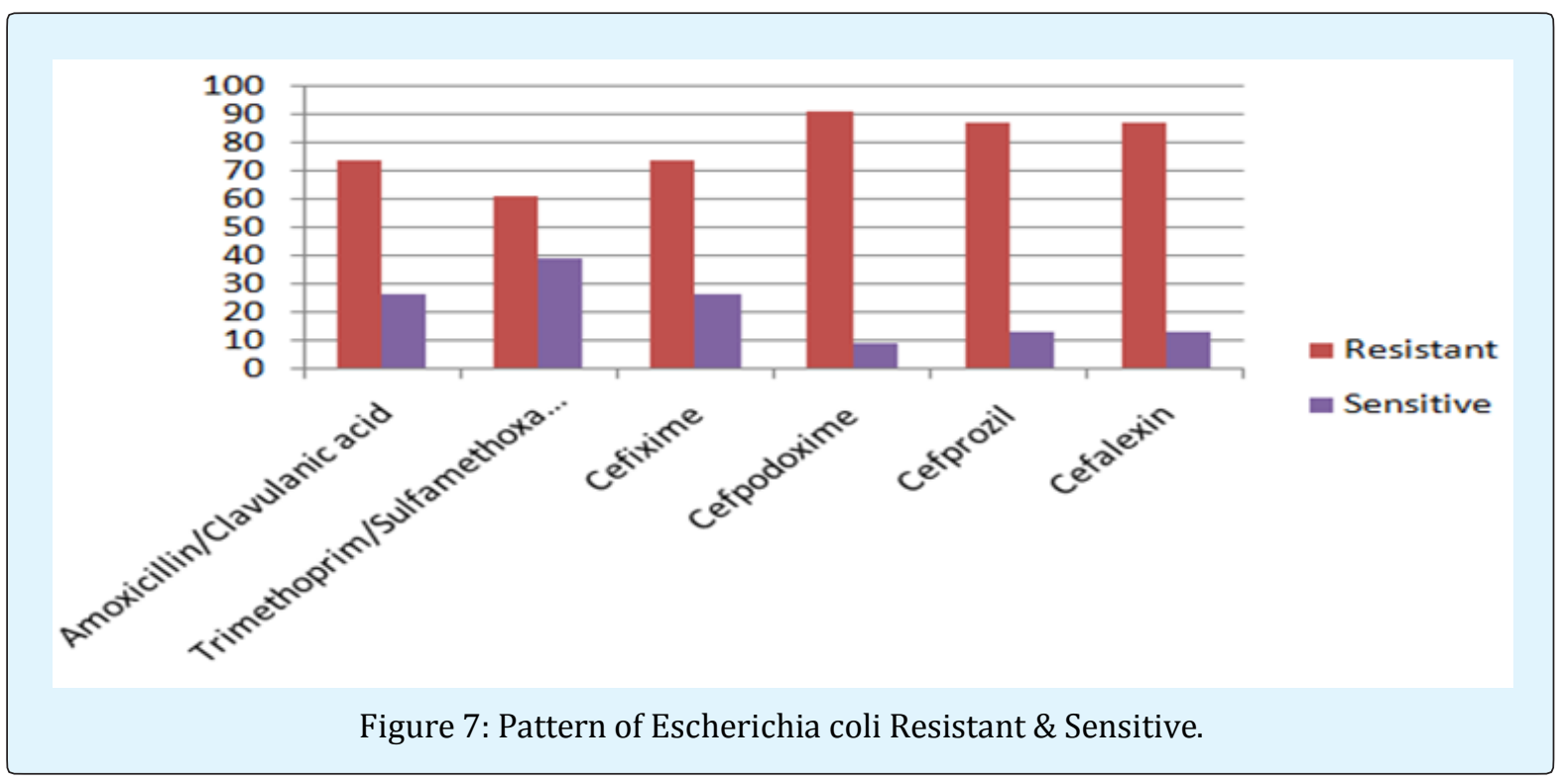

Total Isolates: 20

\begin{tabular}{|c|c|c|c|c|}
\hline Antibiotics & R (No.) & R (\%) & S (No.) & S (\%) \\
\hline Amoxicillin/Clavulanic acid & 10 & 50.0 & 10 & 50.0 \\
\hline Trimethoprim/Sulfamethoxazole & 10 & 50.0 & 10 & 50.0 \\
\hline Cefixime & 06 & 30.0 & 14 & 70.0 \\
\hline Cefpodoxime & 18 & 90.0 & 02 & 10.0 \\
\hline Cefprozil & 12 & 60.0 & 08 & 40.0 \\
\hline Cefalexin & 14 & 70.0 & 06 & 30.0 \\
\hline
\end{tabular}

Table 15: Percentage of Resistant \& Susceptibility of isolated Klebsiella pneumoniae to tested commonly use of oral antibiotics: [>5 TO 12 YEARS].

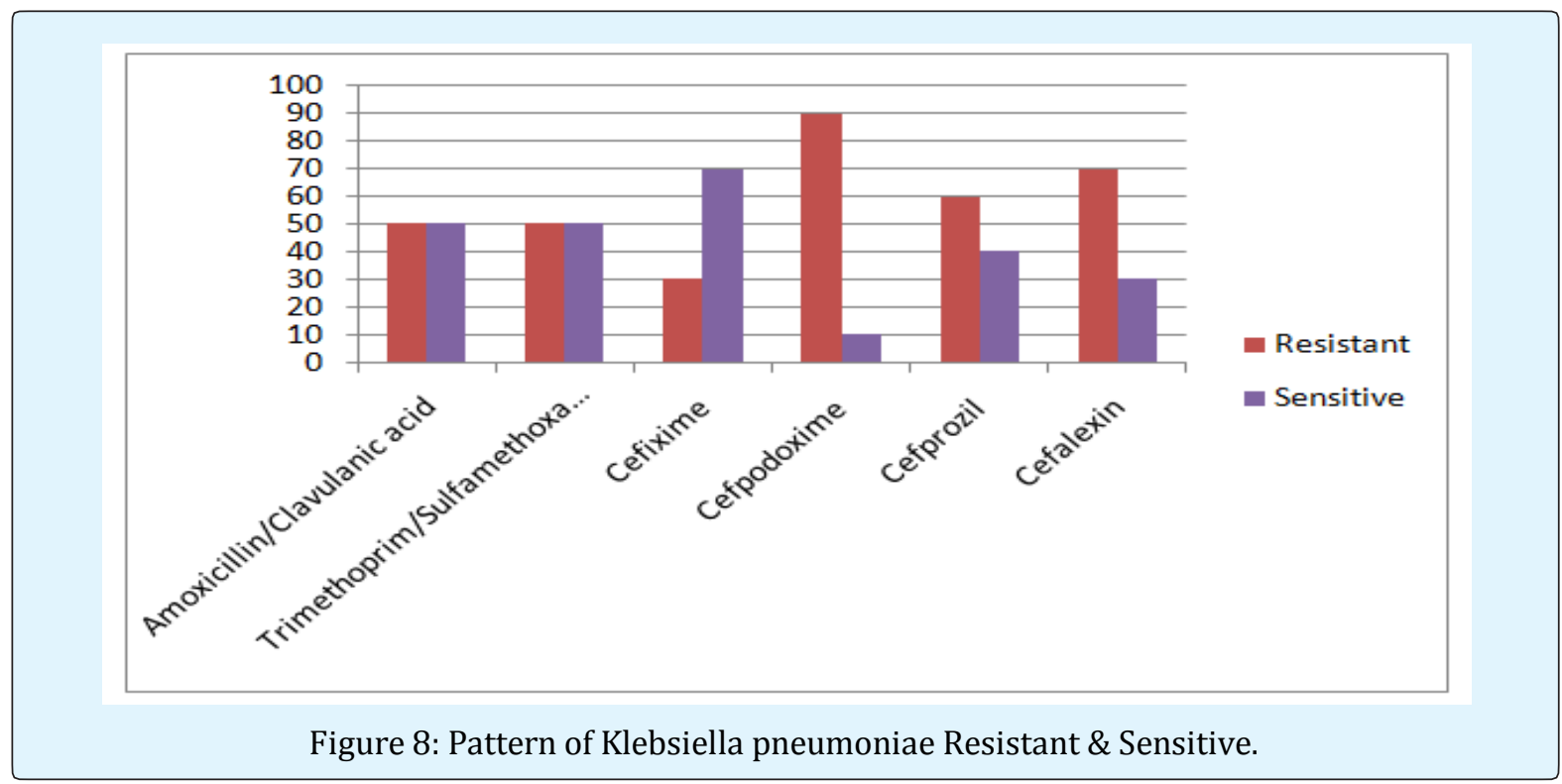


Total Isolates: 06

\begin{tabular}{|c|c|c|c|c|}
\hline Antibiotics & R (No.) & R (\%) & S (No.) & S (\%) \\
\hline Amoxicillin/Clavulanic acid & 04 & 66.6 & 02 & 33.4 \\
\hline Trimethoprim/Sulfamethoxazole & 02 & 33.4 & 04 & 66.7 \\
\hline Cefixime & 01 & 16.6 & 05 & 83.4 \\
\hline Cefpodoxime & 05 & 83.3 & 01 & 16.7 \\
\hline Cefprozil & 05 & 83.3 & 01 & 16.7 \\
\hline Cefalexin & 05 & 83.3 & 01 & 16.7 \\
\hline
\end{tabular}

Table 16: Percentage of Resistant \& Susceptibility of isolated Others Gram Negative Bacilli to tested commonly use of oral antibiotics: [>5 TO 12 YEARS].

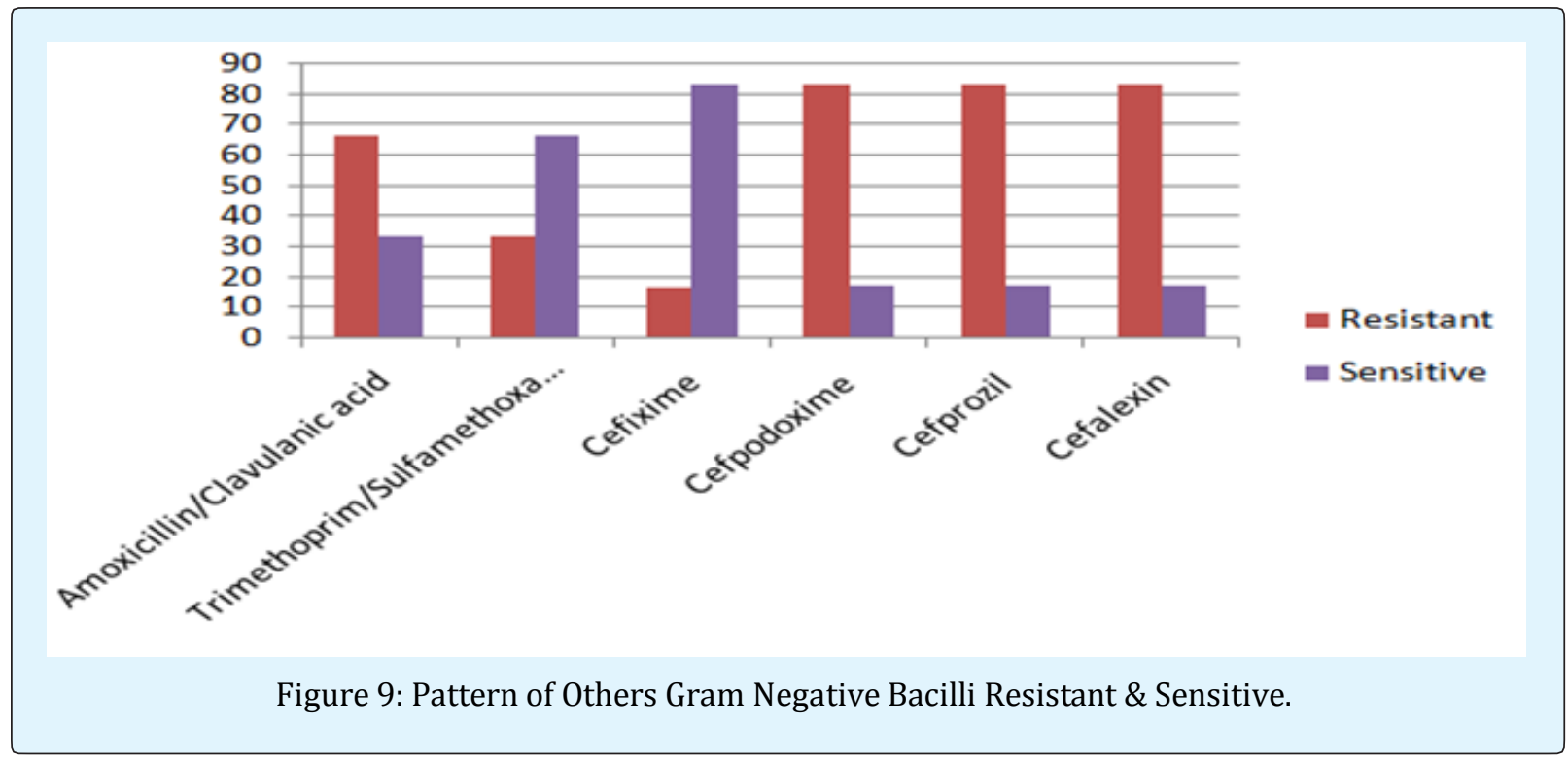

\section{Discussion}

Urinary tract infections are common, potentially serious infection of childhood. Community acquired urinary tract infections (UTI) cause significant illness in the first 2 years of life and are considered as common disease in school and pre-school children [16-18]. Urinary tract infection in children is a significant source of morbidity. It is generally agreed that children with UTI require further investigation and continuing urinary surveillance to minimize future complications.

Escherichia coli is the most common cause of urinary tract infection [19]. Other urinary pathogens include Klebsiella pneumoniae, Enterobacter aerogenes, Citrobacter sp., Pseudomonas aerugenosa, Proteus sp., Enterococcus faecalis, Enterococcus faecalis [20-22]. Our findings are consistent with these reports. In our study confirmed Escherichia coli are major urinary pathogen and urinary tract infection was more common among females than male children.

The Results of the present study indicate a high incidence of microbial resistance to commonly use of oral antibiotics of Amoxicillin/clavulanic acid, Co-trimoxazole, Cefixime, Cefpodoxime, Cefprozil, Cefalexin in urinary tract infections among children (Table 8 to 16) and suggest the physicians to be cautious about treatment with anitibiotics. Knowledge of the local antibiotic resistance helps in guiding antibiotic choice.

Extended-spectrum beta-lactamases (ESBL) are enzymes that confer resistance to most beta-lactam antibiotics, including penicillins, cephalosporins, and the monobactam aztreonam. Infections with ESBL-producing organisms have been associated with poor outcomes. Community and hospital-acquired ESBL-producing Enterobacteriaceae are prevalent worldwide [23]. 


\section{Open Access Journal of Microbiology \& Biotechnology}

Reliable identification of ESBL-producing organisms in clinical laboratories can be challenging, so their prevalence is likely underestimated. Carbapenems are the best antimicrobial agent for infections caused by such organisms.

Beta-lactamases are enzymes that open the beta-lactam ring, inactivating the antibiotic. The first plasmidmediated beta-lactamase in gram-negative bacteria was discovered in Greece in the 1960s. It was named TEM after the patient from whom it was isolated (Temoniera) [24]. Subsequently, a closely related enzyme was discovered and named TEM-2. It was identical in biochemical properties to the more common TEM-1 but differed by a single amino acid with a resulting change in the isoelectric point of the enzyme.

These two enzymes are the most common plasmidmediated beta-lactamases in gram-negative bacteria, including Enterobacteriaceae, Pseudomonas aeruginosa, Haemophilus influenzae, and Neisseria gonorrhoeae. TEM1 and TEM-2 hydrolyze penicillins and narrow spectrum cephalosporins, such as cephalothin or cefazolin. However, they are not effective against higher generation cephalosporins with an oxyimino side chain, such as cefotaxime, ceftazidime, ceftriaxone, or cefepime. Consequently, when these antibiotics were first introduced, they were effective against a broad group of otherwise resistant bacteria. A related but less common enzyme was termed SHV, because sulfhydryl reagents had a variable effect on substrate specificity.

Antibiotic resistance is an important issue affecting public health, and rapid detection in clinical laboratories is essential for the prompt recognition of antimicrobialresistant organisms. Infection-control practitioners and clinicians need the clinical laboratory to rapidly identify and characterise different types of resistant bacteria efficiently to minimise the spread of these bacteria and help to select more appropriate antibiotics. This is particularly true for ESBL-producing bacteria. The epidemiology of ESBL-producing bacteria is becoming more complex with increasingly blurred boundaries between hospitals and the community. E. coli that produce CTX-M $\beta$ lactamases seem to be true community ESBL producers with different behaviours from Klebsiella $s p p$, which produce TEM-derived and SHV-derived ESBLs. These bacteria have become widely prevalent in the community setting in certain areas of the world and they are most likely being imported into the hospital setting.
A recent trend is the emergence of community-onset bloodstream infections caused by ESBL-producing bacteria, especially CTX-M-producing E. coli. These infections are currently rare, but it is possible that, in the near future, clinicians will be regularly confronted with hospital types of bacteria causing infections in patients from the community. $\beta$-lactums contribute a measure class of safer antibiotics. They are widely used as broad spectrum antibiotics for all the type of infections. New generation of antibiotics is predominantly preffered in clinical use. Many more new $\beta$ - lactums are expected for the clinical use and many new $\beta$ - lactums are expected in future. There is a better scope, prosperity for the discovery and development of new and safer $\beta$ lactums.The structure of $\beta$ - lactams,their nature, classification, chemistry to be well studied. $\beta$ - lactums, their mode of action, their bacteriocidal properties and their future growth is seen with new hopes. In this study, in the age group of 1 to 5 years, ESBL found $54.0 \%$ in male child \& $59.5 \%$ in female child and the age group of $>5$ to 12 years, ESBL found $46.7 \%$ in male child and $57.2 \%$ in female child.

This study clearly demonstrates the development of resistance for commonly use of oral antibiotics in children UTI. Different factors are attributable for emergence of resistance mainly include; high consumption of antibiotics, irrational use, incomplete course of therapy, and self-medication by patients, leading to the emergence of resistance and even treatment failures. One major cause of self-medication is poverty. India is an under developed country, people are used to treating themselves without obtaining prescriptions from physicians. The present situation is alarming, because it is not long before common antibiotics, an effective antibiotic would be failed to treat even simple or minor infections. Curtailed follow up of regimen also creates resistance. Generally patients stop their treatment when they feel slight improvement and the microorganisms start adapting the environment rather than get killed. Governments must initiates different educational programs, seminars, workshops in collaboration with the media to make people aware of the consequences of selfmedication, especially with broad- spectrum antibiotics. In addition to this, routine antimicrobial susceptibility testing must be timely performed to determine the current status of resistance against antimicrobial agents (MIC, E test, Disk diffusion method). Otherwise therapy failures may occur which increase the cost of the therapy as well as recovery time from the underlying disease. 


\section{Open Access Journal of Microbiology \& Biotechnology}

\section{Conclusion}

Antimicrobial resistance is a globally ever increasing problem. The emergence and spread of antimicrobial resistance are complex and driven by numerous interconnected factors. The principle causes of microbial resistance are inappropriate, irrational, high consumption, and profligate use of antibiotics. The use of antimicrobials must be restricted and monitored in order to decline the resistance. The present results in increasing commonly use of oral antibiotic resistance trends in UTI patients in children indicate that it is imperative to rationalize the use of antimicrobials and to use these conservatively. Considering the relatively increase rates of UTI and drug resistance observed in this study, continued local, regional, and national surveillance is warranted. Antibiotics should only be issued when prescribed by physicians.

Antibiotic resistance is a growing problem in pediatric urology as highlighted by the significantly increased urinary pathogen resistance to commonly use of oral antibiotics. Poor empiric prescribing practices, lack of urine testing, and nonselective use of prophylaxis exacerbate this problem. However, three small changes in practice patterns may curb the growing resistance rates: use of urine testing in order to only treat when indicated and tailor broad-spectrum therapy as able; selective application of antibiotic prophylaxis to patients; and use of local antiobiograms, particularly pediatric-specific antiobiograms, with inpatient versus outpatient data.

This study will provide novel, clinically important information on the diagnostic features of childhood UTI and the cost effectiveness of a validated prediction rule, to help primary care clinicians improve the efficiency of their diagnostic strategy for UTI in children. Regular monitoring is required to establish reliable information about resistance pattern of urinary pathogens for optimal empirical therapy of patients with UTIs. Finally, we suggest that empirical antibiotic selection should be based on the knowledge of local prevalence of bacterial organisms and antibiotic sensitivities rather than on universal guidelines.

\section{Acknowledgements}

I would like to thank DR. (MRS.) HIMANSHU of the Department of Microbiology; OPJS University; Churu; Rajasthan; India for critical review of the manuscript and suggestions. I express my sincere thanks to Managing Director of Serum Analysis Centre Pvt. Ltd.; Howrah-
711101; West Bengal; India for granting me permission to work in the department and extending all facilities available.

\section{References}

1. Gatermann SG, Borriello P, Murray PR, Funke G (2007) Bacterial infections of the urinary tract. Topley \& Wilson's microbiology \& microbial infections $1^{\text {th }}$ (Edn.), Vol.III, Hodder Arnold Publishers, London, pp: 671-683.

2. Larcombe J (1999) Urinary tract infection in children. BMJ 319: 1173-1175.

3. Shaw KN, Gorelick M, McGowan KL, Yakscoe NM, Schwartz JS (1998) Prevalence of urinary tract infection in febrile young children in the emergency department. Pediatrics 102(2).

4. Bachur R, Harper MB (2001) Reliability of the urinalysis for predicting urinary tract infections in young febrile children. Arch Pediatr Adolesc Med 155(1): 60-65.

5. Twaij M (2000) Urinary tract infection in children: a review of its pathogenesis and risk factors. J R Soc Health 120(4): 220-226.

6. Sharma R, Sharma CL, Kapoor B (2005) Antibacterial resistance: current problems and possible solutions. Indian J Med Sci 59(3): 120-129.

7. Bonev B, Hooper J, Parisot J (2008) Principles of assessing bacterial susceptibility to antibiotics using the agar diffusion method. J Antimicrob Chemother 61(6): 1295-1301.

8. Nweneka CV, Tapha-Sosseh N, Sosa A (2009) Curbing the menace of antimicrobial resistance in developing countries. Harm Reduct J 6: 31.

9. Collee JG, Duguid JP, Fraser AG, Marmion BP, Simmons A (1996) Laboratory strategy in diagnosis of infective syndromes. In: Collee JG, et al. (Eds.), Mackie and McCartney Practical Medical Microbiology $14^{\text {th }}$ (edn), Churchill Livingstone, London, pp: 53-94.

10. Cruickshank R, Duguid JP, Marmion BP (1975) Tests for identification of bacteria. Medical Microbiology $12^{\text {th }}$ (edn), Churchill Livingstone, London, pp: 170189. 
11. Kass EH (1957) Bacteriuria and diagnosis of infections of urinary tract. Arch Intern Med 100(5): 709-714.

12. Monika Cheesbrough (2002) District laboratory Practice in Tropical Countries 2nd (Edn.), Part 2, Cambridge University Press, UK, pp: 132-234.

13. Bauer AW, Kirby WM, Sherris JC, Turck M (1966) Antibiotic susceptibility testing by a standardized single disk method. Am J Clin Pathol 45(4): 493-496.

14. CLSI (2009) Methods for Dilution Antimicrobial Susceptibility Tests for Bacteria that Grow aerobically; Approved Standard 8th (edn), Clinical and Laboratory Standards Institute.

15. CLSI (2009) Performance Standards for Antimicrobial Susceptibility Testing, 20th Informational Supplement, Clinical and Laboratory Standards Institute.

16. Schlager T (2003) Urinary tract infections in infants and children. Infect Dis Clin North Am 17(2): 353365.

17. Wald ER (2004) Cystitis and pyelonephritis. In: Feigin RD, et al. (Eds.), Textbook of Pediatric Infectious Diseases $5^{\text {th }}($ edn), Saunders, Philadelphia, pp: 541553.

18. Fallahzadeh MH, Alamdarlu HM (1999) Prevalence of urinary tract infection in pre-school febrile children. Iranian J of Med Sci 24: 35-39.
19. Esmaeili M (2005) Antibiotics for causative microorganisms of urinary tract infections. Iranian Journal of Pediatric infection 15(2): 163-173.

20. Fluit AC, Mark J, Josef FS, Jacques A, Renu G, et al. (2000) Antimicrobial resistance among urinary tract infection (UTI) isolates in Europe: results from the SENTRY Antimicrobial Surveillance Program 1997. Antonie Van Leeuwenhoek 77(2): 147-152.

21. Jalali M, Asteraki T, Moghadam EE, Kalantar E (2005) Epidemiological study of asymptomatic bacteriuria among nursery school children in Ahwaz, Iran. Afr J Clin Expt Microbiol 6(2): 159-161.

22. Esmaeili M (2005) Antibiotics for causative microorganisms of urinary tract infections. Iranian Journal of Pediatrics 15(2): 165-173.

23. Ben-Ami R, Rodríguez-Baño J, Arslan H, Pitout JD, Quentin C, et al. (2009) A multinational survey of risk factors for infection with extended-spectrum betalactamase-producing enterobacteriaceae in nonhospitalized patients. Clin Infect Dis 49(5): 682690.

24. Bradford PA (2001) Extended-spectrum betalactamases in the 21st century: Characterization, Epidemiology, and Detection of this important resistance threat. Clin Microbiol Rev 14(4): 933-951. 